\title{
Insulated multinozzle system for electrohydrodynamic atomization in the simple-jet mode
}

\author{
L. L. F. Agostinho, ${ }^{1,2}$ C. U. Yurteri, ${ }^{1}$ J. Wartena,${ }^{3}$ S. P. Brouwer, ${ }^{3}$ E. C. Fuchs, ${ }^{2}$ \\ and J. C. M. Marijnissen ${ }^{1,2}$ \\ ${ }^{1}$ Faculty of Applied Sciences, Delft University of Technology, Delft 2628 BL, The Netherlands \\ ${ }^{2}$ Wetsus Centre of Excellence for Sustainable Water Technology, Leeuwarden 8900 CC, The Netherlands \\ ${ }^{3}$ Energy Technology Section, Process and Energy Department, Delft University of Technology, Delft 2628 BL, \\ The Netherlands
}

(Received 24 November 2012; accepted 28 April 2013; published online 16 May 2013)

\begin{abstract}
In this study, a multinozzle electrohydrodynamic atomization device was designed and studied for electrosprays in the simple-jet mode. Results have shown that the proposed device produces droplets with similar diameter in all nozzles, that the electric field creates a unique dispersion of the charged droplets and that it can operate under relatively high humidity conditions. (C) 2013 AIP Publishing LLC. [http://dx.doi.org/10.1063/1.4806977]
\end{abstract}

Electrohydrodynamic atomization (EHDA), shortly electrospraying, is the atomization of a bulk liquid under the influence of electrical forces. ${ }^{1-3}$ The different droplet formation mechanisms in this atomization method, also known as electrospray modes, are mainly related to the flow rate and the characteristics of the electric field. ${ }^{4}$ Many authors have reported about the different modes in electrospray. ${ }^{1,2,5-7}$ Among them, Grace and Marijnissen ${ }^{2}$ showed that they can be divided into two general categories: continuous and noncontinuous modes. The former consists of the simple-jet, the cone-jet, and the ramified jet mode; the latter of the dripping, the microdripping, the spindle, and the intermittent cone-jet mode. Recently, Agostinho et al. ${ }^{8}$ have related the electrospray modes with the different regimes used in non-charged situations to classify the formation of the droplets, i.e., dripping regime, jetting regime, and transition regime. ${ }^{9-11}$ The authors said that the electrospraying modes known as dripping, microdripping, spindle, intermittent cone-jet, and cone-jet mode happen when the flow is adjusted to a level comparable to the dripping regime (low flow rates) and the simple-jet mode happens when the flow rate is adjusted to a level comparable to the transition and jetting regimes (higher flow rates).

EHDA in the so called cone-jet mode is widely used in processes which require droplets in the nano and micrometer size range with narrow size distribution. However, key drawbacks that have hampered its application are, first, the low flow rates and, to a lesser extent, the restrictions on the physical properties of the liquids that can be atomized with this technique. ${ }^{12}$ Good reviews about the different applications of EHDA and their characteristics can be found in the literature. ${ }^{3,13,14}$

Many studies have been done on out-scaling electrospraying in the cone-jet mode. The most explored option is the design of multinozzle devices. Some examples are the works presented by Bocanegra et al., ${ }^{15}$ Deng et al., ${ }^{12}$ and Arnanthigo et $a l .^{4}$ The first authors reported on the construction and operation of a multi-hole electrospray atomizer, bearing up to 37 holes with a packing density (PD) of up to 115 emitters per square centimeter. The second reported the successful operation of a multiplexed electrospray system with a packing density of up to 11547 sources per square centimeter. The third designed a multinozzle electrospray device operating with high precision pumps and using a hydraulic head.

Out-scaling of EHDA by increasing the number of capillary nozzles seems to be a simple way to increase the throughput of these systems. However, factors such as electrical interferences between the neighboring sprays and nonuniformity of the liquid flow rate to all nozzles are problems that have to be overcome. ${ }^{4}$

Increasing the flow rate per nozzle in the cone-jet mode is not an option for out-scaling as the droplet size is a function of this parameter. It is however possible to electrospray in another mode, the simple-jet mode, which operates at much higher flow rate. ${ }^{1,2,6}$ Some information about this mode has been reported in the literature ${ }^{8,16}$ but it is not largely applied because it forms much bigger droplets than the cone-jet mode for the same nozzle geometry. ${ }^{6}$ However, Agostinho et al. ${ }^{17}$ have shown that the application of an electric field in that case can decrease the droplet diameter up to $20 \%$ in relation to the uncharged situation for the jetting regime and up to $50 \%$ in the transition regime, produces droplets with a charge of 5\%-10\% of their Rayleigh limit and can create monodisperse sprays. ${ }^{16}$ Additionally, it also provides dispersion of the droplets after the breakup. ${ }^{17}$ The authors concluded that such mode is a good option for applications which require high throughput with droplets on the hundreds of micrometers range.

Taking into consideration the two options mentioned above one can expect that multinozzle systems operating in the simple-jet mode would provide a very high throughput without requiring a high packing density.

In this work, we designed a multinozzle device for electrospraying in the simple-jet mode. We show that the device proposed can operate in this mode and that the characteristics of each individual nozzle are similar regarding flow per nozzle and produced droplet diameter. Additionally, an insulation layer was applied between the nozzle tip and the counter electrode to allow its operation under high humidity levels without current leakages. The proposed configuration works with the mode which presents the highest flow rate per 


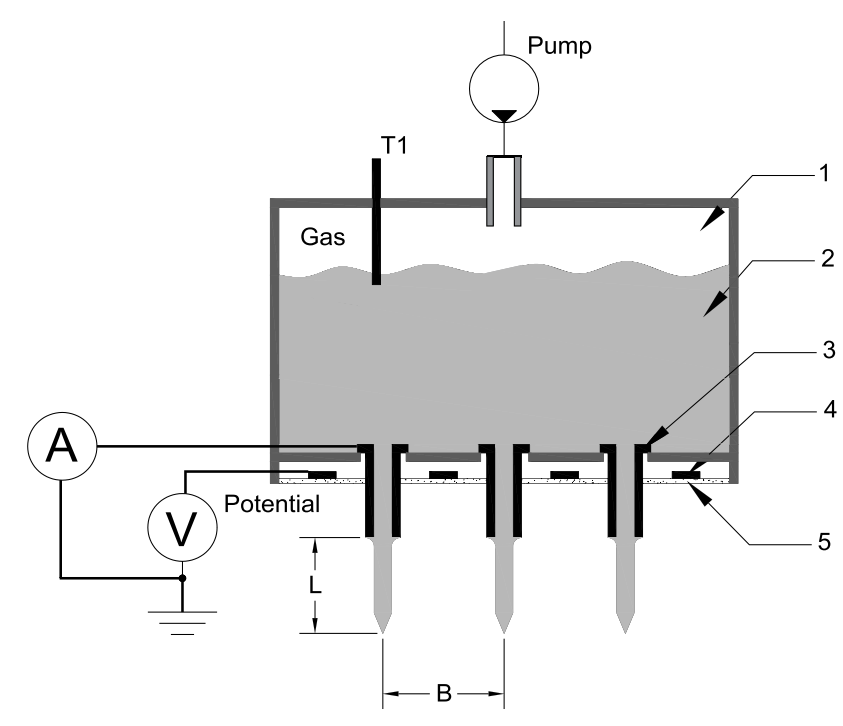

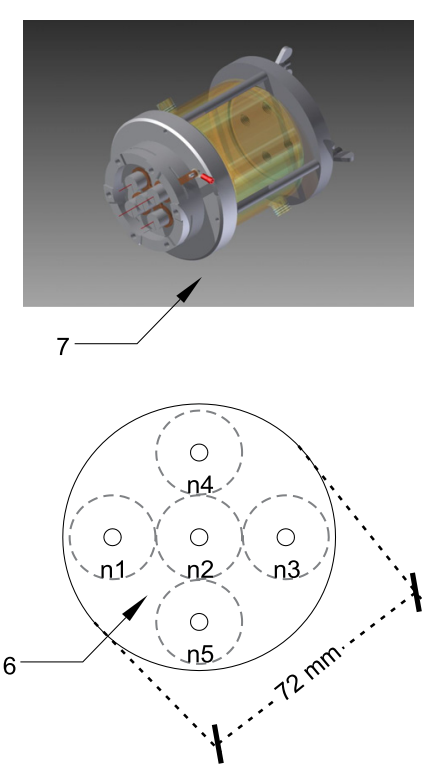

FIG. 1. Multinozzle configuration. (1) Gas column. (2) Liquid column. (3) Metallic nozzles. (4) Counter electrodes (rings). (5) Protective layer (insulation). (6) Multinozzle array. (7) 3D view of the glass heating jacket and the multinozzle body without the insulative layer. The break-up length is represented by $\mathrm{L}$ and the distance between two nozzles $(18 \mathrm{~mm})$ by B. nozzle in EHDA; therefore, it offers very high throughput with very low packing density.

Figure 1 is a representation of the multinozzle device. We chose a nozzle-ring electrospray configuration with the nozzle grounded and the counter electrodes (rings) charged. The device is composed of 4 nozzles arranged in a circular configuration around a central nozzle. The configuration was chosen for its symmetry and to study the influence of neighboring nozzles on a central one. The five nozzles (EFD precision tips $\mathrm{ID}=250 \mu \mathrm{m}$ and $\mathrm{OD}=510 \mu \mathrm{m}$ ) are located in a $36 \mathrm{~mm}$ radius circumference, which gives a PD of $2 \times 10^{3}$ nozzles $\mathrm{m}^{-2}$. Each nozzle is isolated by a cylindrical chamber to avoid possible contact with the counter electrodes. The counter electrodes are copper rings positioned concentrically with each nozzle and placed $1.7 \mathrm{~cm}$ above the nozzle tip. An insulation layer (circuit board, FR-4) is placed in between the nozzle tips and the rings (Figure 1, caption 5) in order to keep the charged rings isolated from other parts of the device (the evaporation chamber).

The liquid used for the experiments was an aqueous solution of $\mathrm{NaCl}$ (Sigma Aldrich) at $35 \mathrm{~g} \mathrm{l}^{-1}$ (physical properties listed in Table I). It was forced through an aperture on the top part of the chamber using a pump (SIMDOS $^{\circledR}$ Model: FEM 1.10 KT.18S). To maintain a constant and equal flow rate through each nozzle and decrease possible oscillations from the pump, a gas column was kept above the liquid/gas interface inside the pre-heating chamber. This chamber got pressurized (1.2-1.6 bar) due to the action of the pump.

In all experiments, the high potential was supplied to the rings by a FUG HCP 35-35000 DC power supply while nozzles and liquid were grounded. To measure the electric

TABLE I. Physical properties of the liquid.

\begin{tabular}{lccccc}
\hline \hline Liquid & $\mu(\mathrm{Pa} \times \mathrm{s})$ & $\rho\left(\mathrm{kg} \times \mathrm{m}^{3}\right)$ & $\varepsilon_{\mathrm{r}}$ & $\mathrm{K}\left(\mathrm{S} \times \mathrm{m}^{-1}\right)$ & $\gamma\left(\mathrm{N} \times \mathrm{m}^{-1}\right)$ \\
\hline Dwater & $1.00 \times 10^{-3}$ & $1.00 \times 10^{3}$ & $8.01 \times 10^{1}$ & $1.20 \times 10^{-3}$ & $7.19 \times 10^{-2}$ \\
$\mathrm{NaCl}$ aq. & $9.21 \times 10^{-4}$ & $1.05 \times 10^{3}$ & $7.35 \times 10^{1}$ & $4.5 \times 10^{0}$ & $7.3 \times 10^{-2}$ \\
\hline \hline
\end{tabular}

current through the spray, an electrometer (Fluke 8846A 6.5) was connected between the ground and the nozzles. The body of the multinozzle device (7) was made of Polyoximethylene (DELRIN) because of its high resistivity, resistance to corrosion and thermal resistance. The pressurized chamber was made of glass to allow visualization of the liquid/gas interface.

To define the dimensions and general characteristics of the device, a theoretical analysis of its electric field was initially made using $\mathrm{Comsol}^{\circledR}$ Multiphysics 4.2. The sprays and their similarities were investigated by measuring the droplet size and breakup length (L) at each nozzle using high speed imaging (Photron SA1). After being recorded, the pictures were analyzed using Image ${ }^{\circledR}$ software and a homemade routine developed in MATLAB ${ }^{\circledR}$.

The experiments were done for three different flow rates $\left(300,360\right.$, and $420 \mathrm{ml} \mathrm{h}^{-1}$ nozzle $\left.{ }^{-1}\right)$. For each flow rate, the potential difference between nozzle and counter electrode was varied between 0 and $8 \mathrm{kV}$. The electric current for each applied potential was defined as the average value measured each $2 \mathrm{~s}$ during $10 \mathrm{~min}$ of uninterrupted spray time. The spray symmetry, droplet size distribution, and droplet dispersion were investigated with respect to the applied flow rate and the potential difference by taking photographs of the spray from large distances $(4 \mathrm{~m})$ using a Canon EOS 550d digital camera with frontal illumination.

A long term experiment was designed to evaluate the effectiveness of the insulation layer which was made to avoid electrical contact between the rings and other parts of the device when the spray operated at very high humidity levels. For that experiment, the multinozzle device was coupled to a closed glass chamber (evaporation chamber, not shown in Figure 1). The influent was pre-heated to $80^{\circ} \mathrm{C}$ and the spray was running uninterruptedly for approximately $13 \mathrm{~h}$. Humidity inside the chamber was measured using a humidity probe. The electric current, inlet temperature, and the humidity inside the chamber were monitored online during the whole experiment. The experiment was repeated for 4 different potentials $(2,4,6$, and $7 \mathrm{kV})$. 

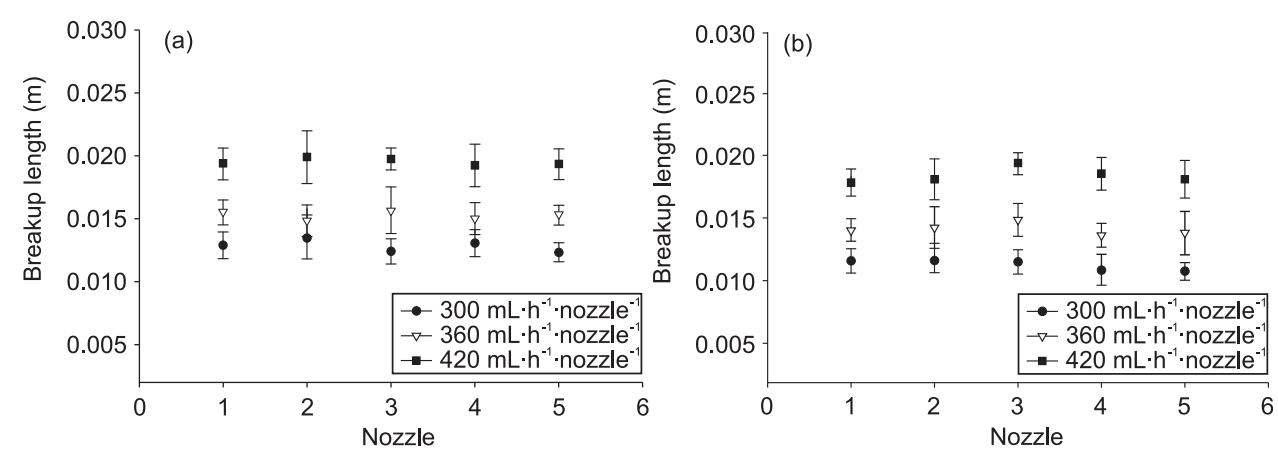

FIG. 2. Breakup length (L) in each nozzle at different flow rates $\left(300 \mathrm{ml} \mathrm{h}^{-1}\right.$ nozzle $^{-1}, 360 \mathrm{ml} \mathrm{h}^{-1}$ nozzle $^{-1}$, and $420 \mathrm{ml} \mathrm{h}^{-1}$ nozzle $^{-1}$ ) for 0 (3a) and $3 \mathrm{kV}$ (3b). Error bars represent the standard error of each measurement.
Figures 2 and 3 show the spray breakup length and droplet average diameter, respectively, in each nozzle, obtained by high speed imaging. Each data point is an average measurement of 20000 images recorded at 1000 frames per second of the spray in each nozzle. The experiments were done in duplicate for three different flow rates $\left(300,360\right.$, and $420 \mathrm{~mL} \mathrm{~h}^{-1}$ nozzle $\left.{ }^{-1}\right)$ and two different potentials $(0$ and $3 \mathrm{kV})$.

Figure 2 shows a constant breakup length for each nozzle which indicates a similar flow rate through each of them for both situations (with and without the application of the electric potential). The similar breakup length found at each nozzle for the uncharged situation $(\Phi=0)$ is an indication that the flow rate is equally distributed over them. The fact that the same behavior is seen for the charged situation $(\Phi=3 \mathrm{kV})$ is a good indication that the magnitude and characteristics of the electric field are similar in each nozzle.

As expected $L$ increases with increasing flow rate ${ }^{3,18,19}$ and a small decrease is observed with the application of an electric potential. This reduction is probably caused by the reduction of the jet radius due to the influence of the electric field. ${ }^{20}$ Such behavior is known and was recently reported by Agostinho et al. in a study about the characteristics of the simple-jet mode. ${ }^{17}$

Figure 3 shows similar average droplet diameters for all the nozzles for the different flow rates and potential differences. This is expected as the diameter of the droplets generated under such conditions is a function of the jet radius which also determines the breakup length. ${ }^{3}$ Additionally, the data show a small decrease of the droplet diameter with the application of the electric potential and an increase of the same parameter for higher flow rates.

The influence of the applied potential on the droplet size distribution is shown in Figure 4. It is possible to see that the spray initially presents a relative standard deviation (RSD), i.e., ratio between the standard deviation and the droplet average size, of approximately 0.4 for the 300 and $420 \mathrm{ml} \mathrm{h}^{-1}$ nozzle ${ }^{-1}$ and 0.25 for $360 \mathrm{ml} \mathrm{h}^{-1}$ nozzle $^{-1}$.
After the application of the electric potential $(3 \mathrm{kV})$ the RSD of the three flow rates decreases to $\sim 0.22$. This is an indication that the application of the electric potential improves size distribution, i.e., it favors the production of monodisperse sprays. The plot also shows RSD values comparable for all nozzles.

The multinozzle spray was also studied regarding the dispersion of the produced droplets. Figure 5 is a view of the spray for a constant flow rate $\left(300 \mathrm{ml} \mathrm{h}^{-1}\right.$ nozzle $\left.{ }^{-1}\right)$ and different electric potentials $(0$ to $7.5 \mathrm{kV})$. The influence of the applied potential is immediately seen because the dispersion of the droplets is enhanced as it increases. The figure also shows that the droplets produced by the outside nozzles are similarly dispersed in relation to the central nozzle for all the applied potentials. This can be related to the previously mentioned facts that the spray behaves identically for all nozzles.

The dispersion of electrosprayed droplets in the simplejet mode was already studied and reported in a previous work for single nozzles (Agostinho et al. ${ }^{17}$ ). In this work it is mentioned that the dispersion is a function of the applied potential and flow rate, i.e., the dispersion angle increases exponentially for a single flow rate if the electrical potential is increased. A very similar behavior can be expected for multinozzles as such systems are normally configured to exclude, as much as possible, inter-nozzle influences.

In the proposed configuration and with the given experimental conditions, the central nozzle produces a stable spray with a symmetric configuration. The four outside sprays presented a slight outward deviation due to edge effects (see Figure 5). This indicates that such system can be extended indefinitely if extra nozzles are placed in the same pattern, using the same inter-nozzle distance and at the same overall conditions.

Computer modeling of the electric field for the presented configuration already showed that the outside sprays (nozzles) have only a small influence on the magnitude of the axial component of the electric field around the central nozzle, i.e.,
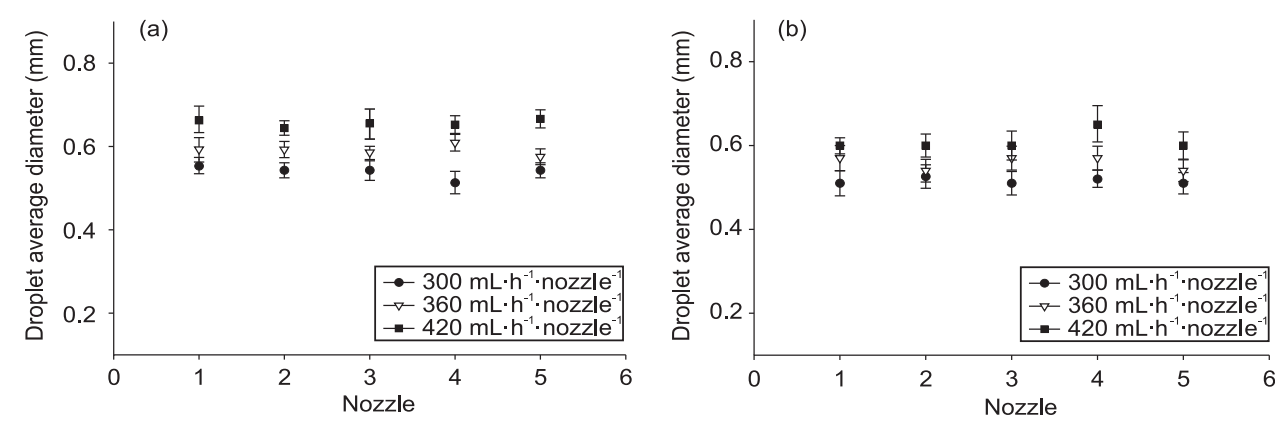

FIG. 3. Droplet average diameter in each nozzle at different flow rates $\left(300 \mathrm{ml} \mathrm{h}^{-1}\right.$ nozzle $^{-1}, 360 \mathrm{ml} \mathrm{h}^{-1}$ nozzle $^{-1}$, and $420 \mathrm{ml} \mathrm{h}^{-1}$ nozzle $^{-1}$ ) for 0 (3a) and $3 \mathrm{kV}$ (3b). Error bars represent the standard error of each measurement. 


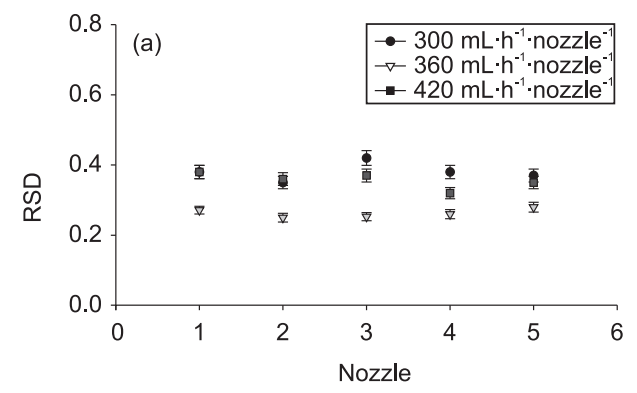

less than $1 \%$, while the radial component decreases up to $7 \%$. This influence is visible in the central nozzle spray (see Figure 5(b)). However, further research is needed to find the minimal nozzle distance, as a function of the liquid properties, applied voltage (for a certain configuration), and flow rate.

In some experiments performed without the insulation layer we observed that under these conditions some droplets, after being sprayed, were returning attracted by the charged rings. These droplets were eventually short circuiting the region between nozzle and ring and high current peaks (some times sparks) could be detected which ultimately interrupted the functioning of the spray. The same problem happened much faster when the multinozzle device was coupled to an evaporation chamber. But in this case it was also due to the accumulation of condensed water vapor in the region between nozzle and ring.

To verify whether the presence of an insulation layer could guarantee a stable operation of the multinozzle system we ran the electrospray inside an evaporation chamber for $13 \mathrm{~h}$ with a flow rate of $360 \mathrm{ml} \mathrm{h}^{-1}$ nozzle ${ }^{-1}$ and a potential difference of $7 \mathrm{kV}$.

Figure 6 shows that the presence of the insulation layer effectively avoids high oscillations of the electric current during the operation of the spray. The oscillation of the electric current observed during the first $200 \mathrm{~min}$ (red curve) was

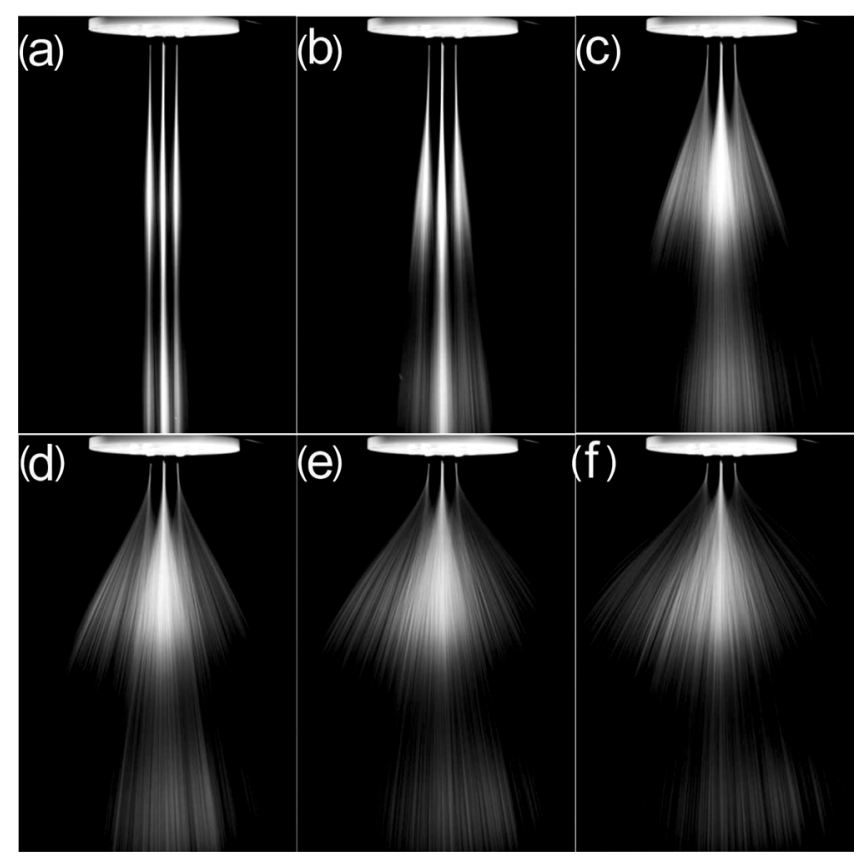

FIG. 5. Overview of the multinozzle spray. The flow was stabilized at $300 \mathrm{mlh}^{-1}$ nozzle $^{-1}$ and the potential was increased from $0(5 \mathrm{a})$ to $7.5 \mathrm{kV}$ (5f) in steps of $1.5 \mathrm{kV}$.
FIG. 4. Relative standard deviation of the generated droplets in each nozzle at different flow rates $\left(300 \mathrm{ml} \mathrm{h}^{-1}\right.$ nozzle ${ }^{-1}$, $360 \mathrm{ml} \mathrm{h}^{-1}$ nozzle $^{-1}$, and $420 \mathrm{ml} \mathrm{h}^{-1}$ nozzle $^{-1}$ ) for $0(3 \mathrm{a})$ and $3 \mathrm{kV}(3 \mathrm{~b})$. Error bars are calculated error based on the distributions.

most probably due to the unstable temperature of the liquid influent during the same time interval (black curve). After this period the electric current stabilized around $\sim 2 \mu \mathrm{A}$. Other experiments were performed for different flow rates (300 and $420 \mathrm{ml} \mathrm{h}^{-1}$ nozzle $^{-1}$ ) and electrical potentials ( 3 and $5 \mathrm{kV}$ ); and similar results were obtained.

We present a multinozzle spraying device having 4 nozzles placed in a circular array around a central nozzle electrospraying in the simple-jet mode. Electrospraying in this mode with only 5 nozzles has provided throughputs of $2.21 \mathrm{~h}^{-1}$ with rather low packing density. ${ }^{12}$ Additionally, the presence of an insulation layer between nozzle tips and counter electrodes allowed a stable operation of the device under high relative humidity levels. Droplet size and jet breakup length measurements proved that all nozzles are operating at very similar conditions. Long distance images showed that the electric potential causes a significant dispersion of the droplets. Results indicate that such a configuration can be a good option for processes which require high throughput, droplets with diameter in the sub-millimeter range and aim at the evaporation of the influent after atomization.

This work was performed in the TTIW-cooperation framework of Wetsus, centre of excellence for sustainable water technology (www.wetsus.nl). Wetsus is funded by the Dutch Ministry of Economic Affairs. The authors would like to thank the participants of the research theme salt for the fruitful discussions and their financial support.

${ }^{1}$ M. Cloupeau and B. Prunet-Foch, J. Aerosol Sci. 25(6), 1021-1036 (1994). ${ }^{2}$ J. M. Grace and J. C. M. Marijnissen, J. Aerosol Sci. 25(6), 1005-1019 (1994).

${ }^{3}$ N. Ashgriz, Handbook of Atomization and Sprays (Springer, New York, NY, 2011). 
${ }^{4}$ Y. Arnanthigo, C. U. Yurteri, G. Biskos, J. C. M. Marijnissen, and A. Schmidt-Ott, Powder Technol. 214, 382-387 (2011).

${ }^{5}$ A. Jaworek and A. Krupa, J. Aerosol Sci. 30(7), 975 (1999).

${ }^{6}$ M. Cloupeau and B. Prunet-Foch, J. Electrost. 25(2), 165-184 (1990).

${ }^{7}$ J. Zeleny, Phys. Rev. 10(1), 1 (1917).

${ }^{8}$ L. L. F. Agostinho, J. Wartena, E. C. Fuchs, C. U. Yurteri, and J. C. M. Marijnissen, paper presented at the European Aerosol Conference, Manchester, UK, 4-9 September 2011.

${ }^{9}$ C. Clanet and J. C. Lasheras, J. Fluid Mech. 383, 307-326 (1999).

${ }^{10}$ B. Ambravaneswaran, H. J. Subramani, S. D. Phillips, and O. A. Basaran, Phys. Rev. Lett. 93(3), 034501 (2004).

${ }^{11}$ B. Ambravaneswaran, S. D. Phillips, and O. A. Basaran, Phys. Rev. Lett. 85(25), 5332-5335 (2000).

${ }^{12}$ W. Deng, C. M. Waits, B. Morgan, and A. Gomez, J. Aerosol Sci. 40(10), 907-918 (2009).
${ }^{13}$ K. B. Geerse, "Applications of Electrospray: from People to Plants," Ph.D. dissertation, Delft University of Technology, Delft, Netherlands, 2003.

${ }^{14}$ C. U. Yurteri, R. P. A. Hartman, and J. C. M. Marijnissen, KONA Powder Part. J. 28, 24 (2010).

${ }^{15}$ R. Bocanegra, D. Galán, M. Márquez, I. G. Loscertales, and A. Barrero, J. Aerosol Sci. 36(12), 1387-1399 (2005).

${ }^{16}$ L. L. F. Agostinho, C. U. Yurteri, E. C. Fuchs, and J. C. M. Marijnissen, Appl. Phys. Lett. 100(24), 244105 (2012).

${ }^{17}$ L. L. F. Agostinho, G. Tamminga, C. U. Yurteri, S. P. Brouwer, E. C. Fuchs, and J. C. M. Marijnissen, Phys. Rev. E 86, 066317 (2012).

${ }^{18}$ J. Eggers and E. Villermaux, Rep. Prog. Phys. 71(3), 036601 (2008).

${ }^{19}$ S. P. Lin, Breakup of Liquid Sheets and Jets (Cambridge University Press, 2010).

${ }^{20}$ R. T. Collins, M. T. Harris, and O. A. Basaran, J. Fluid Mech. 588, 75-129 (2007). 